\title{
The Annual American Men's Internet Survey of Behaviors of Men Who Have Sex With Men in the United States: 2017 Key Indicators Report
}

Maria Zlotorzynska, MPH, PhD; Cera Cantu, MPH; Ramona Rai, MPH; Patrick Sullivan, DVM, PhD; Travis Sanchez, MPH, DVM

Department of Epidemiology, Rollins School of Public Health, Emory University, Atlanta, GA, United States

\section{Corresponding Author:}

Maria Zlotorzynska, MPH, $\mathrm{PhD}$

Department of Epidemiology

Rollins School of Public Health

Emory University

1518 Clifton Rd NE

Atlanta, GA, 30317

United States

Phone: 14047278799

Email: mzlotor@emory.edu

\section{Abstract}

The American Men's Internet Survey (AMIS) is an annual Web-based behavioral survey of men who have sex with men (MSM) who live in the United States. This Rapid Surveillance Report describes the fifth cycle of data collection (July 2017 to November 2017: AMIS 2017). The key indicators are the same as those previously reported for past AMIS cycles (December 2013 to May 2014: AMIS 2013; November 2014 to April 2015: AMIS 2014; September 2015 to April 2016: AMIS 2015; and September 2016 to February 2017: AMIS 2016). The AMIS methodology has not substantively changed since AMIS 2016. The MSM were recruited from a variety of websites using banner advertisements and email blasts. Additionally, participants from AMIS 2016 who agreed to be recontacted for future research were emailed a link to AMIS 2017. Men were eligible to participate if they were aged $\geq 15$ years, resided in the United States, provided a valid US zone improvement plan code, and reported ever having sex with a man or identified as gay or bisexual. The analysis was limited to those who reported having oral or anal sex with a male partner in the past 12 months. We examined demographic and recruitment characteristics using multivariable regression modeling $(P<.05)$ stratified by the participants' self-reported HIV status. The AMIS 2017 round of data collection resulted in 10,049 completed surveys from MSM representing every US state, Puerto Rico, and Guam. Participants were mainly non-Hispanic white, over the age of 40 years, living in the Southern United States and urban areas, and recruited from geospatial social networking websites. The plurality $(4485 / 10,049,44.6 \%)$ of participants was in the 40 years and older age group, followed by the youngest age group, 15 to 24 years $(2726 / 10,049,27.1 \%)$. Self-reported HIV prevalence was $9.6 \%(964 / 10,049)$. Compared with HIV-negative or unknown-status participants, HIV-positive participants were more likely to have had anal sex without a condom with a male partner in the past 12 months (adjusted odds ratio [aOR] 2.21, 95\% CI 1.86-2.63) and more likely to have had anal sex without a condom with a serodiscordant or an unknown-status partner (aOR 3.13, 95\% CI 2.71-3.62). The reported use of marijuana in the past 12 months was higher among HIV-positive participants than HIV-negative or unknown status participants (aOR 1.29, 95\% CI 1.09-1.51). The reported use of methamphetamines and other illicit substances in the past 12 months was higher among HIV-positive participants than HIV-negative or unknown status participants (aOR 5.57, 95\% CI 4.38-7.09 and aOR 1.93, 95\% CI 1.65-2.27, respectively). Most HIV-negative or unknown status participants (7330/9085, 80.7\%) reported ever taking an HIV test previously, and 60.6\% (5504/9085) reported undergoing HIV testing in the past 12 months. HIV-positive participants were more likely to report testing and diagnosis of sexually transmitted infections than HIV-negative or unknown status participants (aOR 2.85, 95\% CI 2.46-3.31 and aOR 2.73, 95\% CI 2.29-3.26, respectively).

(JMIR Public Health Surveill 2020;6(2):e16847) doi: 10.2196/16847

\section{KEYWORDS}

HIV; internet; men who have sex with men; sexually transmitted infections: surveillance; survey 


\section{Introduction}

The American Men's Internet Survey (AMIS) is an annual Web-based behavioral survey of men who have sex with men (MSM) who live in the United States. AMIS was developed to produce timely data from large-scale monitoring of behavior trends among MSM recruited on the Web. It was designed to complement the Centers for Disease Control and Prevention's National HIV Behavioral Surveillance (NHBS) system, which collects data on MSM in major US cities every 3 years through venue-based recruitment [1]. An increasing number of MSM are meeting sexual partners through the internet and may have different patterns of sexual risk and HIV testing behaviors compared with MSM recruited through physical venues. AMIS is able to generate annual snapshots of behaviors in a large sample of internet-using MSM with broad geographic diversity as a supplement to venue-based studies, such as the NHBS system. We were also able to collect, update, and share state-level data with public health authorities to inform issues of local relevance by using AMIS.

The methods and past AMIS cycle data (AMIS 2013, AMIS 2014, AMIS 2015, and AMIS 2016) have been previously published [2-5].

This supplemental report has updated the existing information with data collected in AMIS 2017. The methods in AMIS 2017 have not changed from the previously published methods, unless otherwise noted. An in-depth analysis and discussion of multiyear trends for indicators reported herein has been published and includes data for the first 4 cycles of AMIS (AMIS 2013 to AMIS 2016) [6].

\section{Methods}

\section{Recruitment and Enrollment}

Similar to the previous year's recruitment process, AMIS participants were recruited through convenience sampling from a variety of websites using banner advertisements or email blasts to members of the website (hereafter referred to generically as ads). For AMIS 2017, data were collected from July 2017 to November 2017. The survey was not incentivized. Data on the number of clicks on all banner ads were obtained directly from the websites. Men who clicked on the ads were taken directly to the survey website hosted on a secure server administered by SurveyGizmo (Boulder, Colorado). Recruitment was also done by emailing participants from the previous cycle of AMIS (AMIS 2016) who consented to be recontacted for future studies. To be eligible for the survey, participants had to be aged $\geq 15$ years, be a cisgender male, reside in the United States, and report that they either had oral or anal sex with a male partner at least once in the past or identify as gay or bisexual (hereafter referred to as MSM). Persons who were aged $<15$ years or refused to provide their age were not asked any other screening questions. MSM who met the eligibility criteria and consented to participate in the study started the Web-based survey immediately. The full questionnaire for AMIS 2017 is presented in Multimedia Appendix 1.
Several data cleaning steps were performed on the raw dataset of eligible responses to obtain the final analysis dataset, in the same manner as in previous AMIS cycles [2-6]. Briefly, these steps were as follows: deduplication; limiting to surveys deemed successful, ie, observations with no missing values for the first question of at least two consecutive sections; limiting to participants who reported having oral or anal sex with a male partner in the past 12 months; and zone improvement plan (ZIP) code validation. These steps are further described in detail.

First, to deduplicate survey responses, demographic data for near-complete $(>70 \%)$ survey responses with nonunique internet protocol addresses were compared, and responses that showed a $100 \%$ match for all characteristics were considered to be duplicate responses. Only the observation with highest survey completion was retained. The dataset was, then, limited to those surveys that were deemed successful. Finally, the dataset was restricted to include participants who reported having oral or anal sex in the past 12 months and who provided a valid US ZIP code. ZIP codes were validated in the same manner as done in AMIS 2016 [5]. Valid US ZIP codes were those that could be matched to the ZIP code of county crosswalk files created by the US Department of Housing and Urban Development [7]. Any ZIP codes that could not be matched to this list were, then, hand-validated by checking against the ZIP code locator tool in the US Postal Service website [8]. ZIP codes that could not be found were classified as invalid.

\section{Measures and Analyses}

For the AMIS 2017 analyses, participants were categorized as either AMIS 2016 participants who took the survey again or new participants from the website/app based on the target audience and purpose: gay social networking $(n=2)$, gay general interest $(n=1)$, general social networking $(n=4)$, and geospatial social networking $(n=2)$. Recruitment outcomes and demographic characteristics for the AMIS 2017 participants are presented in Tables 1 and 2, and thereafter, they are recategorized according to their original source of recruitment. We did not provide the names of the websites/apps to preserve operator and client privacy, particularly when a category has only 1 operator. Participants whose data were eligible, unduplicated, and successful and who provided consent, reported having male-male sex in the past 12 months, and provided a valid US ZIP code were included in analyses of participant characteristics and behavior.

To facilitate comparisons, the key indicators and analytic approach used in AMIS were designed to mirror those used by the NHBS system [9]. Population density was defined in the same manner as defined in AMIS 2016 and was based on the National Center for Health Statistics Rural-Urban classification scheme for counties [10]. The self-reported HIV status was categorized as HIV-positive, and HIV-negative or unknown status, consistent with surveillance reports produced by the NHBS system [9]. In total, 3 substance use behaviors in the past 12 months were assessed: use of nonprescribed marijuana, use of methamphetamines, and use of any illicit drug other than marijuana or methamphetamines. All other indicators assessed remained unchanged from AMIS 2016 [5]. 
The analysis methods for AMIS 2017 did not substantively differ from those previously published but are repeated in this report for clarity. Overall, chi-square tests were used to identify whether participant characteristics differed significantly among recruitment sources. Multivariable logistic regression modeling was used to determine significant differences in behaviors based on the self-reported HIV status while controlling for race/ethnicity, age group, NHBS city residency, and type of recruitment website. The metropolitan statistical areas included in the NHBS system in 2017 were as follows: Atlanta, Georgia; Boston, Massachusetts; Chicago, Illinois; Dallas, Texas; Denver, Colorado; Detroit, Michigan; Houston, Texax; Los Angeles, California; Memphis, Tennessee; Miami, Florida;
Nassau-Suffolk, New York; New Orleans, Louisiana, New York City, New York; Newark, New Jersey; Philadelphia, Philadelphia; Portland, Oregon; San Diego, California; San Francisco, California; San Juan, Puerto Rico; Seattle, Washington; Virginia Beach-Norfolk, Virginia; and Washington, District of Columbia. HIV testing behaviors were only examined among those who did not report that they were HIV positive, and these data were presented in participant characteristics. The multivariable logistic regression results were presented as Wald chi-square $P$ values to denote an independently significant difference in the behavior for each subgroup compared with a reference group. Statistical significance was set at $P<.05$.

Table 1. Recruitment outcomes for the American Men's Internet Survey, United States, 2017.

\begin{tabular}{|c|c|c|c|c|c|c|}
\hline Recruitment outcomes & Total & $\begin{array}{l}\text { Gay social network- } \\
\text { ing }(n=2)^{\mathrm{a}}\end{array}$ & $\begin{array}{l}\text { General gay inter- } \\
\text { est }(n=1)^{\text {a }}\end{array}$ & $\begin{array}{l}\text { General social net- } \\
\text { working }(\mathrm{n}=4)^{\mathrm{a}}\end{array}$ & $\begin{array}{l}\text { Geospatial social } \\
\text { networking }(n=2)^{a}\end{array}$ & $\begin{array}{l}\text { AMIS }^{\mathrm{b}} 2016 \\
\text { participants }\end{array}$ \\
\hline Clicked ad, N & 210,505 & 4700 & 421 & 191,958 & 13,426 & $\mathrm{~N} / \mathrm{A}^{\mathrm{c}}$ \\
\hline Screened $^{\mathrm{d}}, \mathrm{n}(\%)$ & $69,002(32.78)$ & $3136(66.72)$ & 394 (93.59) & $51,472(26.81)$ & $12,306(91.66)$ & 1694 \\
\hline Ineligible $^{\mathrm{e}}, \mathbf{n}(\%)$ & $40,299(58.40)$ & $461(14.70)$ & 247 (62.69) & $36,970(71.83)$ & $2507(20.37)$ & $114(6.73)$ \\
\hline Not $>15$ years of age ${ }^{f}$ & $5297(13.14)$ & $34(7.38)$ & $2(0.81)$ & $5025(13.59)$ & $230(9.17)$ & $6(5.26)$ \\
\hline Not male ${ }^{f}$ & $21,409(53.13)$ & $345(74.84)$ & $59(23.89)$ & $19,084(51.62)$ & $1832(73.08)$ & $89(78.07)$ \\
\hline $\begin{array}{l}\text { Not MSM }{ }^{\mathrm{g}} \text { ever or not } \\
\text { identifying as gay/bi- } \\
\text { sexual }^{\mathrm{f}}\end{array}$ & $39,528(98.09)$ & $414(89.80)$ & $68(27.53)$ & $36,746(99.39)$ & $2191(87.40)$ & $109(95.61)$ \\
\hline Nonresident ${ }^{\mathrm{f}}$ & $19,997(49.62)$ & $280(60.74)$ & $236(95.55)$ & $17,619(47.66)$ & $1800(71.80)$ & $62(54.39)$ \\
\hline Eligible $^{\mathrm{e}}, \mathrm{n}(\%)$ & $28,703(41.60)$ & $2675(85.30)$ & $147(37.31)$ & $14,502(28.17)$ & $9799(79.63)$ & $1580(93.27)$ \\
\hline Consented $^{\mathrm{h}}, \mathrm{n}(\%)$ & $21,731(75.71)$ & $2065(77.20)$ & $129(87.76)$ & $10,483(72.29)$ & $7578(77.33)$ & $1476(93.42)$ \\
\hline Unduplicated $^{\mathrm{i}}, \mathrm{n}(\%)$ & $18,346(84.42)$ & $1874(90.75)$ & $120(93.02)$ & $8328(79.44)$ & $6682(88.18)$ & $1342(90.92)$ \\
\hline Success $^{\mathrm{j}}, \mathrm{n}(\%)$ & $11,159(60.83)$ & $1398(74.60)$ & $95(79.17)$ & $4298(51.61)$ & $4170(62.41)$ & $1198(89.27)$ \\
\hline $\begin{array}{l}\text { MSM in the past } 12 \\
\text { months }{ }^{\mathrm{k}}, \mathrm{n}(\%)\end{array}$ & $10,113(90.63)$ & $1305(93.35)$ & $86(90.53)$ & $3675(85.50)$ & $3953(94.80)$ & 1094 (91.32) \\
\hline Valid ZIP code $^{\mathrm{m}}, \mathrm{n}(\%)$ & 10,049 (99.37) & $1293(99.08)$ & $85(98.84)$ & $3648(99.27)$ & $3931(99.44)$ & $1092(99.82)$ \\
\hline
\end{tabular}

${ }^{\mathrm{a}}$ Refers to the number of websites or apps in this category.

${ }^{b}$ AMIS: American Men's Internet Survey.

${ }^{\mathrm{c}} \mathrm{N} / \mathrm{A}$ : not applicable.

${ }^{\mathrm{d}}$ Proportion of total participants who clicked the ad, including those who started the screening questionnaire.

${ }^{\mathrm{e}}$ Proportion of total participants screened. Participants who did not complete the screening questionnaire were considered ineligible.

${ }^{\mathrm{f}}$ Proportion of total ineligible participants, including those who did not respond to the question.

${ }^{\mathrm{g}}$ MSM: men who have sex with men.

${ }^{\mathrm{h}}$ Proportion of eligible participants.

${ }^{\mathrm{i}}$ Proportion of participants who consented. Deduplication removes participants who were marked as duplicates using the internet protocol address and demographic data matching.

${ }^{\mathrm{j}}$ Proportion of unduplicated participants. Success removes participants who did not pass the test for survey completeness.

${ }^{\mathrm{k}}$ Proportion of successes.

lZIP: zone improvement plan.

${ }^{\mathrm{m}}$ Proportion of men who had sex with men in the past 12 months. Valid US ZIP codes were those that could be matched to the ZIP code for county crosswalk files created by the US Department of Housing and Urban Development. Any ZIP codes that could not be matched to this list were then hand-validated by checking against the ZIP code locator tool in the US Postal Service website. ZIP codes that could not be found were classified as invalid. 
Table 2. Characteristics of men who have sex with men in the American Men's Internet Survey by recruitment type, United States, 2017.

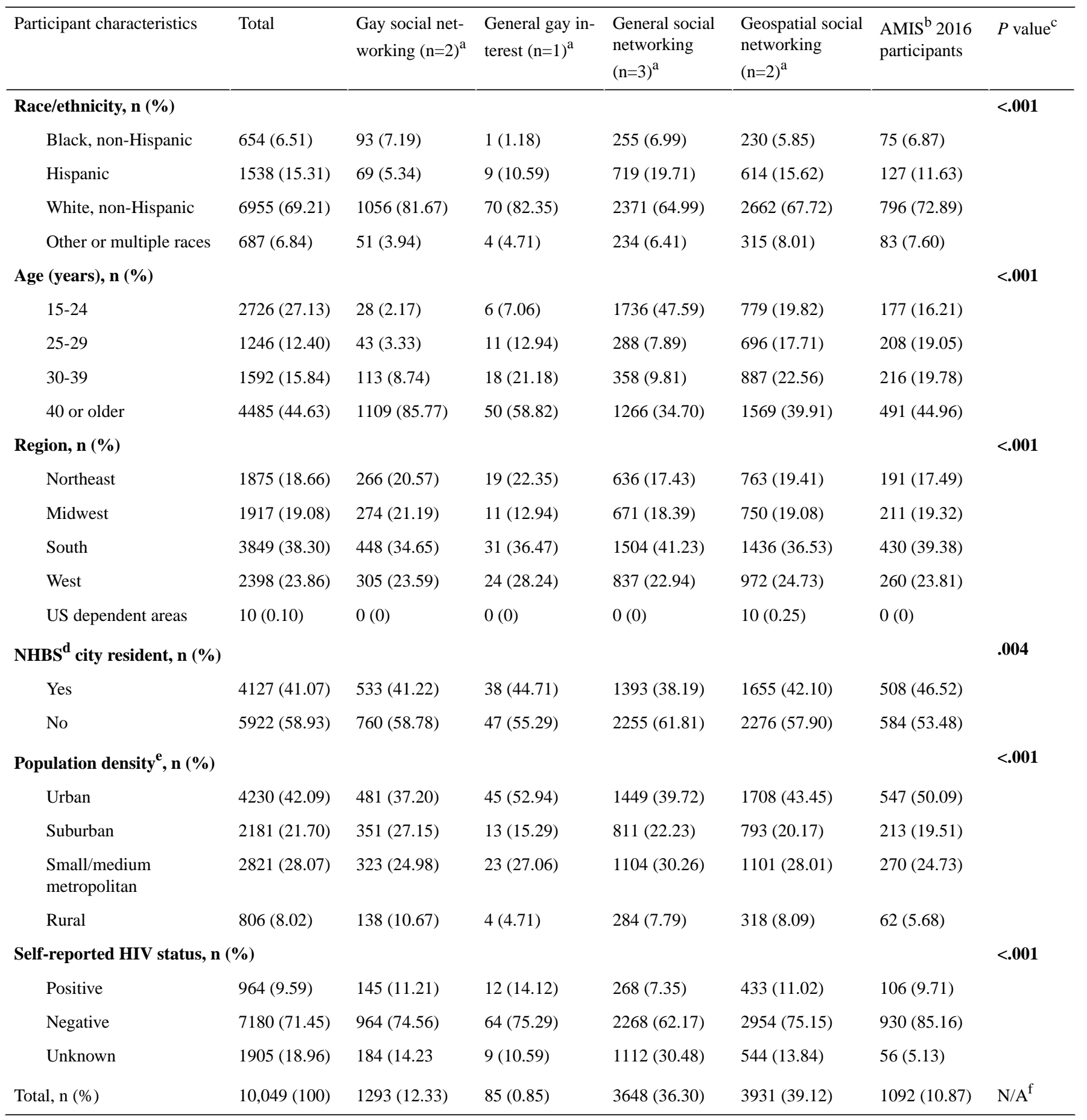

${ }^{\mathrm{a}}$ Refers to the number of websites or apps in this category

${ }^{b}$ AMIS: American Men's Internet Survey.

${ }^{\mathrm{c}} \mathrm{A}$ chi-square test for the difference in characteristics between recruitment types.

${ }^{\mathrm{d}}$ NHBS: National HIV Behavioral Surveillance.

${ }^{\mathrm{e}}$ The National Center for Health Statistics urban/rural category could not be assigned for 10 participants living in US territories.

${ }^{\mathrm{f}}$ Not applicable.

\section{Results}

\section{Recruitment Outcomes}

AMIS 2017 was conducted from July 2017 to November 2017 and resulted in 210,505 persons clicking on the ads and landing on the study's recruitment page (Table 1). Most persons who clicked on the ads were from general networking websites $(191,958 / 210,505,91.1 \%)$. Of the 3713 participants who completed the AMIS 2016 survey and were emailed links to the AMIS 2017 survey, 45.6\% (1694/3713) clicked on the link. About one-third (32.8\%) of all participants who landed on the study page started the screening process and $41.6 \%$ of them 
were eligible. The most common reason for ineligibility was not ever having male-male sex or not identifying as gay or bisexual. Three-quarter $(75.7 \%)$ of participants who were eligible consented to participate in the survey. A total of 3385 $(15.6 \%)$ surveys were likely from duplicate participants. Among unduplicated surveys, $60.8 \%$ were considered successful. Most successful surveys were from men who reported having sex with another male in the past 12 months (90.6\%). Almost all of these surveys $(10,049 / 10,113,99.4 \%)$ provided a valid US ZIP code. Overall, the completion rate was $4.8 \%$, with an analytical sample consisting of 10,049 surveys from 210,505 clicks.

\section{Participant Characteristics}

In total, $69.2 \%(6955 / 10,049)$ of the participants included in this report were non-Hispanic white and $44.6 \%$ were $\geq 40$ years of age $(4485 / 10,049)$; the most common region of residence was the South followed by the West (Table 2). Participants were recruited from all US states, and there were at least 100 participants each from 29 states and the District of Columbia (Figure 1). About 4 in $10(4127 / 10,049,41.1 \%)$ participants resided in an NHBS city and about the same proportion $(4230 / 10,049,42.1 \%)$ lived in an urban county. Overall, $9.6 \%$ $(964 / 10,049)$ of participants were HIV positive, $71.5 \%$ (7180/10,049) were HIV negative, and 19.0\% (1905/10,049) had an unknown HIV status. All participant characteristics differed significantly based on the recruitment source (Table 2).

Figure 1. The number of men who have sex with men who participated in the American Men's Internet Survey (AMIS) by state, 2017.

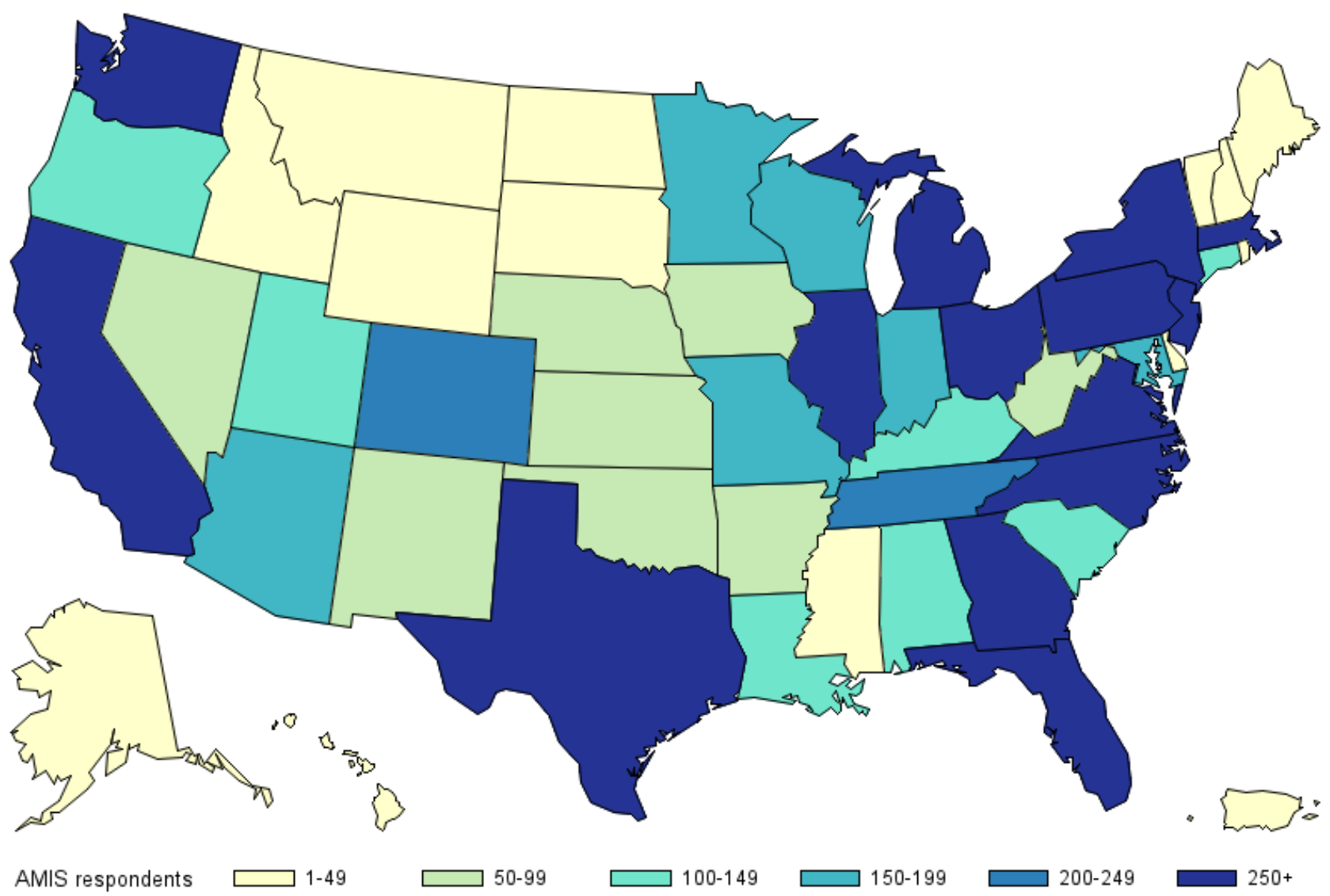

\section{Sexual Behaviors}

Around two-third $(6761 / 10,049,67.3 \%)$ of participants reported having anal sex without a condom with another male in the past 12 months and about one-fifth $(2135 / 10,049,21.3 \%)$ reported doing so with a partner of a discordant or an unknown HIV status (Table 3). Compared with HIV-negative or unknown status participants, those who were HIV positive were significantly more likely to report anal intercourse without a condom (adjusted odds ratio [aOR] 2.21, 95\% CI 1.86-2.63), including with male partners who were of a discordant or an unknown status (aOR 3.13, 95\% CI 2.71-3.62). Stratified by the serostatus group, anal intercourse without a condom differed significantly by race/ethnicity (HIV-negative or unknown status participants only), age group (HIV-negative or unknown status participants), and recruitment website (HIV-negative or unknown status participants only). Anal intercourse without a condom with partners of a discordant or an unknown HIV status differed significantly by age and residence in an NHBS city for HIV-negative or unknown status participants only and race/ethnicity for both HIV-negative or unknown status participants and HIV-positive status participants. 
Table 3. Sexual behaviors with male partners of men who have sex with men in the American Men's Internet Survey, United States, 2017.

\begin{tabular}{|c|c|c|c|c|c|}
\hline \multirow[t]{3}{*}{ Participant characteristics } & \multirow[t]{3}{*}{ Participants (N) } & \multicolumn{4}{|c|}{ Sexual behaviors with male partners in the past 12 months } \\
\hline & & \multicolumn{2}{|c|}{ Anal intercourse without a condom } & \multicolumn{2}{|c|}{$\begin{array}{l}\text { Anal intercourse without a condom with } \\
\text { a partner of a discordant or an unknown } \\
\text { HIV status }\end{array}$} \\
\hline & & $\mathrm{n}(\%)$ & $P$ value $^{\mathrm{a}}$ & $\mathrm{n}(\%)$ & $P$ value $^{\mathrm{a}}$ \\
\hline HIV positive & 964 & $781(81.01)$ & $<.001^{\mathrm{b}}$ & $408(42.32)$ & $<.001^{\mathrm{b}}$ \\
\hline \multicolumn{6}{|l|}{ Race/ethnicity } \\
\hline Black, non-Hispanic & 138 & $102(73.91)$ & .07 & $39(28.26)$ & .01 \\
\hline Hispanic & 139 & $115(82.73)$ & .54 & $56(40.29)$ & .66 \\
\hline White, non-Hispanic & 617 & $511(82.82)$ & $\operatorname{Ref}^{\mathrm{a}}$ & $292(47.33)$ & $\operatorname{Ref}^{\mathrm{a}}$ \\
\hline Other or multiple races & 53 & $42(79.25)$ & .70 & $18(33.96)$ & .47 \\
\hline \multicolumn{6}{|l|}{ Age (years) } \\
\hline $15-24$ & 39 & $29(74.36)$ & .09 & $22(56.41)$ & .16 \\
\hline $25-29$ & 78 & $70(89.74)$ & .05 & $38(48.72)$ & .55 \\
\hline $30-39$ & 172 & $151(87.79)$ & .28 & $86(50.00)$ & .75 \\
\hline 40 or older & 675 & $531(78.67)$ & $\operatorname{Ref}^{\mathrm{a}}$ & $262(38.81)$ & $\operatorname{Ref}^{\mathrm{a}}$ \\
\hline \multicolumn{6}{|l|}{ NHBS $^{c}$ city resident } \\
\hline Yes & 454 & 377 (83.04) & .11 & $187(41.19)$ & .95 \\
\hline No & 510 & $404(79.22)$ & $\operatorname{Ref}^{\mathrm{a}}$ & $221(43.33)$ & $\operatorname{Ref}^{\mathrm{a}}$ \\
\hline \multicolumn{6}{|l|}{ Recruitment type } \\
\hline Gay social networking & 157 & $119(75.80)$ & .16 & $80(50.96)$ & .12 \\
\hline General gay interest & 12 & $11(91.67)$ & .40 & $6(50.00)$ & .80 \\
\hline General social networking & 332 & $261(78.61)$ & $\operatorname{Ref}^{\mathrm{a}}$ & $126(37.95)$ & $\operatorname{Ref}^{\mathrm{a}}$ \\
\hline Geospatial social networking & 462 & $389(84.20)$ & .98 & $195(42.21)$ & .34 \\
\hline HIV negative or unknown status & 9085 & $5980(65.82)$ & $\operatorname{Ref}^{b}$ & $1727(19.01)$ & $\operatorname{Ref}^{b}$ \\
\hline \multicolumn{6}{|l|}{ Race/ethnicity } \\
\hline Black, non-Hispanic & 516 & $332(64.34)$ & .34 & $129(25.00)$ & .007 \\
\hline Hispanic & 1399 & $953(68.12)$ & .008 & $288(20.59)$ & .85 \\
\hline White, non-Hispanic & 6338 & $4186(66.05)$ & $\operatorname{Ref}^{\mathrm{a}}$ & $1161(18.32)$ & $\operatorname{Ref}^{\mathrm{a}}$ \\
\hline Other or multiple races & 634 & $398(62.78)$ & .04 & $121(19.10)$ & .23 \\
\hline \multicolumn{6}{|l|}{ Age (years) } \\
\hline $15-24$ & 2687 & $1665(61.97)$ & $<.001$ & $476(17.72)$ & .02 \\
\hline $25-29$ & 1168 & $849(72.69)$ & $<.001$ & $235(20.12)$ & .45 \\
\hline $30-39$ & 1420 & $1042(73.38)$ & $<.001$ & $311(21.90)$ & .02 \\
\hline 40 or older & 3810 & $2424(63.62)$ & $\operatorname{Ref}^{\mathrm{a}}$ & $705(18.50)$ & $\operatorname{Ref}^{\mathrm{a}}$ \\
\hline \multicolumn{6}{|l|}{ NHBS $^{c}$ city resident } \\
\hline Yes & 3673 & $2464(67.08)$ & .10 & $746(20.31)$ & .047 \\
\hline No & 5412 & $3516(64.97)$ & $\operatorname{Ref}^{\mathrm{a}}$ & $981(18.13)$ & $\operatorname{Ref}^{\mathrm{a}}$ \\
\hline \multicolumn{6}{|l|}{ Recruitment type } \\
\hline Gay social networking & 1268 & $723(57.02)$ & $<.001$ & $247(19.48)$ & .42 \\
\hline General gay interest & 102 & $68(66.67)$ & .98 & $18(17.65)$ & .57 \\
\hline
\end{tabular}




\begin{tabular}{|c|c|c|c|c|c|}
\hline \multirow[t]{3}{*}{ Participant characteristics } & \multirow[t]{3}{*}{ Participants (N) } & \multicolumn{4}{|c|}{ Sexual behaviors with male partners in the past 12 months } \\
\hline & & \multicolumn{2}{|c|}{ Anal intercourse without a condom } & \multicolumn{2}{|c|}{$\begin{array}{l}\text { Anal intercourse without a condom with } \\
\text { a partner of a discordant or an unknown } \\
\text { HIV status }\end{array}$} \\
\hline & & $\mathrm{n}(\%)$ & $P$ value $^{\mathrm{a}}$ & $\mathrm{n}(\%)$ & $P$ value $^{\mathrm{a}}$ \\
\hline General social networking & 4022 & $2588(64.35)$ & $\operatorname{Ref}^{\mathrm{a}}$ & $748(18.60)$ & $\operatorname{Ref}^{\mathrm{a}}$ \\
\hline Geospatial social networking & 3688 & $2597(70.42)$ & $<.001$ & $713(19.33)$ & .74 \\
\hline
\end{tabular}

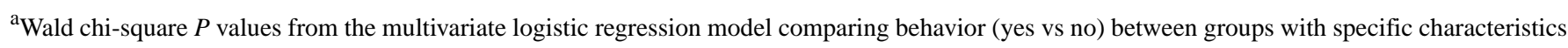
and a reference group (Ref).

${ }^{\mathrm{b}}$ Wald chi-square $P$ values from the multivariate logistic regression model comparing behavior (yes vs no) among HIV-positive participants and HIV-negative or unknown-serostatus participants. Model controlled for race/ethnicity, age, NHBS system city residency, and recruitment type.

${ }^{\mathrm{c}}$ NHBS: National HIV Behavioral Surveillance.

\section{Substance Use Behaviors}

In total, $27.6 \%(2775 / 10,049)$ of participants reported using marijuana, $5.9 \% \quad(363 / 10,049)$ reported using methamphetamines, and $20.8 \%(2086 / 10,049)$ reported using other illicit substances in the past 12 months (Table 4). Compared with HIV-negative or unknown status participants, HIV-positive participants were significantly more likely to report the use of marijuana (aOR 1.29, 95\% CI 1.09-1.51), methamphetamines (aOR 5.57, 95\% CI 4.38-7.09), and other illicit substances (aOR 1.93, 95\% CI 1.65-2.27) in the past 12 months. Among HIV-positive participants, the use of marijuana varied significantly by NHBS city residency, and the use of methamphetamines varied significantly by the recruitment website. In this group, the use of other illicit substances varied significantly by race/ethnicity and residence in an NHBS city. Additionally, the use of marijuana, methamphetamines, and other illicit substances differed significantly by age among HIV-negative or unknown status participants. In this group, the use of marijuana and other illicit substances differed significantly by race/ethnicity and residence in an NHBS city, and the use of other illicit substances differed significantly by the recruitment website. 
Table 4. Substance use behaviors of men who have sex with men in the American Men's Internet Survey, United States, 2017.

\begin{tabular}{|c|c|c|c|c|c|c|c|}
\hline \multirow[t]{2}{*}{ Participant characteristics } & \multirow[t]{2}{*}{ Participants (N) } & \multicolumn{2}{|c|}{ Used marijuana } & \multicolumn{2}{|c|}{ Used methamphetamines } & \multicolumn{2}{|c|}{ Used other substance(s) } \\
\hline & & $\mathrm{n}(\%)$ & $P$ value $^{\mathrm{a}}$ & $\mathrm{n}(\%)$ & $P$ value $^{\mathrm{a}}$ & $\mathrm{n}(\%)$ & $P$ value $^{\mathrm{a}}$ \\
\hline HIV positive & 964 & $255(26.45)$ & $.002^{\mathrm{b}}$ & $136(14.11)$ & $<.001^{\mathrm{b}}$ & $274(28.42)$ & $<.001^{\mathrm{b}}$ \\
\hline \multicolumn{8}{|l|}{ Race/ethnicity } \\
\hline Black, non-Hispanic & 138 & $28(20.29)$ & .16 & $10(7.25)$ & .09 & $23(16.67)$ & .003 \\
\hline Hispanic & 139 & $41(29.50)$ & .24 & $17(12.23)$ & .77 & $40(28.78)$ & .74 \\
\hline White, non-Hispanic & 617 & $172(27.88)$ & $\operatorname{Ref}^{\mathrm{a}}$ & $100(16.21)$ & $\operatorname{Ref}^{\mathrm{a}}$ & $192(31.12)$ & $\operatorname{Ref}^{\mathrm{a}}$ \\
\hline Other or multiple races & 53 & $10(18.87)$ & .35 & $5(9.43)$ & .74 & $15(28.30)$ & .58 \\
\hline \multicolumn{8}{|l|}{ Age (years) } \\
\hline $15-24$ & 39 & $12(30.77)$ & .76 & $5(12.82)$ & .89 & $9(23.08)$ & .41 \\
\hline $25-29$ & 78 & $22(28.21)$ & .95 & $8(10.26)$ & .33 & $26(33.33)$ & .36 \\
\hline $30-39$ & 172 & $62(36.05)$ & .20 & $35(20.35)$ & .10 & $68(39.53)$ & .07 \\
\hline 40 or older & 675 & $159(23.56)$ & $\operatorname{Ref}^{\mathrm{a}}$ & $88(13.04)$ & $\operatorname{Ref}^{\mathrm{a}}$ & $171(25.33)$ & $\operatorname{Ref}^{\mathrm{a}}$ \\
\hline \multicolumn{8}{|l|}{ NHBS $^{c}$ city resident } \\
\hline Yes & 454 & $135(29.74)$ & .02 & $67(14.76)$ & .27 & $146(32.16)$ & .006 \\
\hline No & 510 & $120(23.53)$ & $\operatorname{Ref}^{\mathrm{a}}$ & $69(13.53)$ & $\operatorname{Ref}^{\mathrm{a}}$ & $128(25.10)$ & $\operatorname{Ref}^{\mathrm{a}}$ \\
\hline \multicolumn{8}{|l|}{ Recruitment type } \\
\hline Gay social networking & 157 & $31(19.75)$ & .76 & $11(7.01)$ & .02 & $33(21.02)$ & .09 \\
\hline General gay interest & 12 & $2(16.67)$ & .41 & $0(0.00)$ & $\mathrm{N} / \mathrm{A}^{\mathrm{d}}$ & $5(41.67)$ & .37 \\
\hline $\begin{array}{l}\text { General social network- } \\
\text { ing }\end{array}$ & 332 & $92(27.71)$ & $\operatorname{Ref}^{\mathrm{a}}$ & $40(12.05)$ & $\operatorname{Ref}^{\mathrm{a}}$ & $86(25.90)$ & $\operatorname{Ref}^{\mathrm{a}}$ \\
\hline $\begin{array}{l}\text { Geospatial social net- } \\
\text { working }\end{array}$ & 462 & $129(27.92)$ & .26 & $84(18.18)$ & $<.001$ & $149(32.25)$ & .66 \\
\hline HIV negative or unknown status & 9085 & $2520(27.74)$ & $\operatorname{Ref}^{b}$ & $227(2.50)$ & $\operatorname{Ref}^{b}$ & $1812(19.94)$ & $\operatorname{Ref}^{\mathrm{b}}$ \\
\hline \multicolumn{8}{|l|}{ Race/ethnicity } \\
\hline Black, non-Hispanic & 516 & $132(25.58)$ & .40 & $11(2.13)$ & .27 & $73(14.15)$ & .001 \\
\hline Hispanic & 1399 & $456(32.59)$ & .33 & $34(2.43)$ & .51 & $322(23.02)$ & .002 \\
\hline White, non-Hispanic & 6338 & $1703(26.87)$ & $\operatorname{Ref}^{\mathrm{a}}$ & $152(2.40)$ & $\operatorname{Ref}^{\mathrm{a}}$ & $1259(19.86)$ & $\operatorname{Ref}^{\mathrm{a}}$ \\
\hline Other or multiple races & 634 & $166(26.18)$ & .046 & $22(3.47)$ & .08 & $113(17.82)$ & .22 \\
\hline \multicolumn{8}{|l|}{ Age (years) } \\
\hline $15-24$ & 2687 & $1016(37.81)$ & $<.001$ & $35(1.30)$ & .002 & $597(22.22)$ & .09 \\
\hline $25-29$ & 1168 & $394(33.73)$ & $<.001$ & $25(2.14)$ & .51 & $297(25.43)$ & $<.001$ \\
\hline $30-39$ & 1420 & $444(31.27)$ & .048 & $55(3.87)$ & .001 & $377(26.55)$ & $<.001$ \\
\hline 40 or older & 3810 & $666(17.48)$ & $\operatorname{Ref}^{\mathrm{a}}$ & $112(2.94)$ & $\operatorname{Ref}^{\mathrm{a}}$ & $541(14.20)$ & $\operatorname{Ref}^{\mathrm{a}}$ \\
\hline \multicolumn{8}{|l|}{ NHBS $^{c}$ city resident } \\
\hline Yes & 3673 & 1097 (29.87) & $<.001$ & $103(2.80)$ & .56 & $823(22.41)$ & $<.001$ \\
\hline No & 5412 & $1423(26.29)$ & $\operatorname{Ref}^{\mathrm{a}}$ & $124(2.29)$ & $\operatorname{Ref}^{\mathrm{a}}$ & 989 (18.27) & $\operatorname{Ref}^{\mathrm{a}}$ \\
\hline \multicolumn{8}{|l|}{ Recruitment type } \\
\hline Gay social networking & 1268 & $240(18.93)$ & .66 & 48 (3.79) & .10 & $190(14.98)$ & .90 \\
\hline General gay interest & 102 & $25(24.51)$ & .97 & $4(3.92)$ & .88 & $19(18.63)$ & .68 \\
\hline $\begin{array}{l}\text { General social network- } \\
\text { ing }\end{array}$ & 4022 & $1243(30.91)$ & $\operatorname{Ref}^{\mathrm{a}}$ & $57(42)$ & $\operatorname{Ref}^{\mathrm{a}}$ & 767 (19.07) & $\operatorname{Ref}^{\mathrm{a}}$ \\
\hline
\end{tabular}




\begin{tabular}{|c|c|c|c|c|c|c|c|}
\hline \multirow[t]{2}{*}{ Participant characteristics } & \multirow[t]{2}{*}{ Participants $(\mathrm{N})$} & \multicolumn{2}{|c|}{ Used marijuana } & \multicolumn{2}{|c|}{ Used methamphetamines } & \multicolumn{2}{|c|}{ Used other substance(s) } \\
\hline & & $\mathrm{n}(\%)$ & $P$ value $^{\mathrm{a}}$ & $\mathrm{n}(\%)$ & $P$ value $^{\mathrm{a}}$ & $\mathrm{n}(\%)$ & $P$ value ${ }^{\mathrm{a}}$ \\
\hline $\begin{array}{l}\text { Geospatial social net- } \\
\text { working }\end{array}$ & 3688 & $1012(27.44)$ & .80 & $118(3.20)$ & .46 & $835(22.64)$ & .046 \\
\hline
\end{tabular}

${ }^{\text {a } W a l d ~ c h i-s q u a r e ~} P$ values from the multivariate logistic regression model comparing behavior (yes vs no) between groups with specific characteristics and a reference group (Ref).

${ }^{\mathrm{b}}$ Wald chi-square $P$ values from the multivariate logistic regression model comparing behavior (yes vs no) among HIV-positive participants and HIV-negative or unknown-serostatus participants. Model controlled for race/ethnicity, age, National HIV Behavioral Surveillance system city residency, and recruitment type.

${ }^{\mathrm{c}}$ NHBS: National HIV Behavioral Surveillance.

${ }^{\mathrm{d}} \mathrm{N} / \mathrm{A}$ : not applicable.

\section{HIV Testing Behaviors}

HIV testing behaviors were examined among participants who were not HIV positive (Table 5). Most participants (7330/9085, $80.7 \%$ ) were previously tested for HIV infection, and $60.6 \%$
(5504/9085) were tested in the past 12 months. HIV testing behavior, both ever tested and tested in the past 12 months, differed significantly by race/ethnicity, age, residence in an NHBS city, and type of recruitment website.

Table 5. HIV testing behaviors of HIV-negative or unknown-status men who have sex with men in the American Men's Internet Survey, United States, 2017.

\begin{tabular}{|c|c|c|c|c|c|}
\hline \multirow[t]{3}{*}{ Participant characteristics } & \multirow[t]{3}{*}{ Participants (N) } & \multicolumn{4}{|c|}{ HIV testing behaviors } \\
\hline & & \multicolumn{2}{|c|}{ HIV tested, ever } & \multicolumn{2}{|c|}{ HIV tested, past 12 months } \\
\hline & & $\mathrm{n}(\%)$ & $P$ value ${ }^{\mathrm{a}}$ & $\mathrm{n}(\%)$ & $P$ value $^{\mathrm{a}}$ \\
\hline \multicolumn{6}{|l|}{ Race/ethnicity } \\
\hline Black, non-Hispanic & 516 & $445(86.24)$ & .005 & $353(68.41)$ & .01 \\
\hline Hispanic & 1399 & $991(70.84)$ & .09 & $814(58.18)$ & .51 \\
\hline White, non-Hispanic & 6338 & $5244(82.74)$ & $\operatorname{Ref}^{\mathrm{a}}$ & $3814(60.18)$ & Ref \\
\hline Other or multiple races & 634 & $489(77.13)$ & .23 & $398(62.78)$ & .95 \\
\hline \multicolumn{6}{|l|}{ Age (years) } \\
\hline $15-24$ & 2687 & $1478(55.01)$ & $<.001$ & $1210(45.03)$ & $<.001$ \\
\hline $25-29$ & 1168 & $1034(88.53)$ & .05 & $846(72.43)$ & $<.001$ \\
\hline $30-39$ & 1420 & $1310(92.25)$ & $<.001$ & $1032(72.68)$ & $<.001$ \\
\hline 40 or older & 3810 & $3508(92.07)$ & Ref & $2416(63.41)$ & Ref \\
\hline \multicolumn{6}{|l|}{ NHBS $^{b}$ city resident } \\
\hline Yes & 3673 & $3081(83.88)$ & $<.001$ & $2417(65.80)$ & $<.001$ \\
\hline No & 5412 & $4249(78.51)$ & Ref & $3087(57.04)$ & Ref \\
\hline \multicolumn{6}{|l|}{ Recruitment type } \\
\hline Gay social networking & 1268 & $1094(86.28)$ & $<.001$ & $741(58.44)$ & .03 \\
\hline General gay interest & 102 & $94(92.16)$ & .17 & $60(58.82)$ & .24 \\
\hline General social networking & 4022 & $2916(72.50)$ & Ref & $2076(51.62)$ & Ref \\
\hline Geospatial social networking & 3688 & $3222(87.36)$ & $<.001$ & $2623(71.12)$ & $<.001$ \\
\hline Total & 9085 & $7330(80.68)$ & $\mathrm{N} / \mathrm{A}^{\mathrm{c}}$ & $5504(60.58)$ & N/A \\
\hline
\end{tabular}

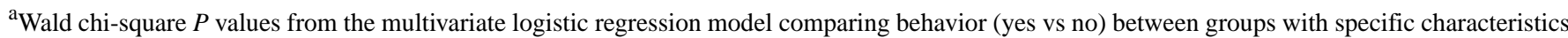
and a reference (Ref) group.

${ }^{\mathrm{b}}$ NHBS: National HIV Behavioral Surveillance.

${ }^{\mathrm{c}} \mathrm{N} / \mathrm{A}$ : not applicable. 


\section{Sexually Transmitted Infection Testing and Diagnosis}

In total, $42.2 \%(4243 / 10,049)$ of participants reported sexually transmitted infection (STI) testing in the past 12 months and just $11.5 \%(1153 / 10,049)$ reported a diagnosis of STI in the past 12 months. Compared with HIV-negative or unknown status participants, HIV-positive participants were significantly more likely to report STI testing (aOR 2.85, 95\% CI 2.46-3.31) and STI diagnosis (aOR 2.73, 95\% CI 2.29-3.26) in the past 12 months (Table 6). The most common STI diagnosis among HIV-positive participants was syphilis (137/964, 14.2\%), followed by gonorrhea $(116 / 964,12.0 \%)$, and chlamydia (112/964, 11.6\%). Chlamydia was the most common STI diagnosis among HIV-negative or unknown status participants
(501/9085, 5.5\%), followed by gonorrhea (481/9085, 5.3\%) and syphilis $(267 / 9085,2.9 \%)$. STI testing significantly differed by age, residence in an NHBS city, and recruitment website among both HIV-positive status participants and HIV-negative or unknown status participants. STI testing also significantly differed by race/ethnicity for HIV-negative or unknown status participants. STI diagnosis significantly differed by race/ethnicity (HIV-negative or unknown status participants only), age (HIV-negative or unknown status participants only), residency in an NHBS city (both HIV-positive status participants and HIV-negative or unknown status participants), and recruitment website (HIV-negative or unknown status participants only). 
Table 6. Sexually transmitted infection testing and diagnosis of men who have sex with men in the American Men's Internet Survey, United States, 2017.

\begin{tabular}{|c|c|c|c|c|c|}
\hline \multirow[t]{3}{*}{ Participant characteristics } & \multirow[t]{3}{*}{ Participants (N) } & \multicolumn{4}{|c|}{$\mathrm{STI}^{\mathrm{a}}$ history in the past 12 months } \\
\hline & & \multicolumn{2}{|c|}{ Tested for any STI } & \multicolumn{2}{|c|}{ Diagnosed with any STI } \\
\hline & & $\mathrm{n}(\%)$ & $P$ value $^{\mathrm{b}}$ & $\mathrm{n}(\%)$ & $P$ value ${ }^{\mathrm{b}}$ \\
\hline HIV positive & 964 & $641(66.49)$ & $<.001^{\mathrm{c}}$ & $236(24.48)$ & $<.001^{\mathrm{c}}$ \\
\hline \multicolumn{6}{|l|}{ Race/ethnicity } \\
\hline Black, non-Hispanic & 138 & $94(68.12)$ & .82 & $43(31.16)$ & .08 \\
\hline Hispanic & 139 & $97(69.78)$ & .75 & $42(30.22)$ & .68 \\
\hline White, non-Hispanic & 617 & $401(64.99)$ & $\operatorname{Ref}^{b}$ & $133(21.56)$ & $\operatorname{Ref}^{\mathrm{b}}$ \\
\hline Other or multiple races & 53 & $39(73.58)$ & .57 & $12(22.64)$ & .31 \\
\hline \multicolumn{6}{|l|}{ Age (years) } \\
\hline $15-24$ & 39 & $25(64.10)$ & .31 & $13(33.33)$ & .52 \\
\hline $25-29$ & 78 & $64(82.05)$ & .03 & $28(35.90)$ & .40 \\
\hline $30-39$ & 172 & $131(76.16)$ & .44 & $60(34.88)$ & .48 \\
\hline 40 or older & 675 & $421(62.37)$ & $\operatorname{Ref}^{\mathrm{b}}$ & $135(20.00)$ & $\operatorname{Ref}^{\mathrm{b}}$ \\
\hline \multicolumn{6}{|l|}{ NHBS $^{d}$ city resident } \\
\hline Yes & 454 & $324(71.37)$ & .005 & $128(28.19)$ & .04 \\
\hline No & 510 & $317(62.16)$ & $\operatorname{Ref}^{b}$ & $108(21.18)$ & $\operatorname{Ref}^{b}$ \\
\hline \multicolumn{6}{|l|}{ Recruitment type } \\
\hline Gay social networking & 157 & $96(61.15$ & $>.99$ & $27(17.20)$ & .29 \\
\hline General gay interest & 12 & $7(58.33$ & .53 & $3(25.00)$ & .79 \\
\hline General social networking & 332 & $193(58.13$ & $\operatorname{Ref}^{b}$ & $61(18.37)$ & $\operatorname{Ref}^{\mathrm{b}}$ \\
\hline $\begin{array}{l}\text { Geospatial social network- } \\
\text { ing }\end{array}$ & 462 & $344(74.46$ & .01 & $145(31.39)$ & .07 \\
\hline HIV negative or unknown status & 9085 & $3602(39.65)$ & $\operatorname{Ref}^{\mathrm{c}}$ & 917 (10.09) & $\operatorname{Ref}^{\mathrm{c}}$ \\
\hline \multicolumn{6}{|l|}{ Race/ethnicity } \\
\hline Black, non-Hispanic & 516 & $248(48.06)$ & .009 & $84(16.28)$ & $<.001$ \\
\hline Hispanic & 1399 & $609(43.53)$ & .26 & $177(12.65)$ & .56 \\
\hline White, non-Hispanic & 6338 & $2409(38.01)$ & $\operatorname{Ref}^{b}$ & $568(8.96)$ & $\operatorname{Ref}^{b}$ \\
\hline Other or multiple races & 634 & $262(41.32)$ & .11 & $68(10.73)$ & .07 \\
\hline \multicolumn{6}{|l|}{ Age (years) } \\
\hline $15-24$ & 2687 & $875(32.56)$ & $<.001$ & $227(8.45)$ & .004 \\
\hline $25-29$ & 1168 & $606(51.88)$ & $<.001$ & $172(14.73)$ & $<.001$ \\
\hline $30-39$ & 1420 & $714(50.28)$ & $<.001$ & $217(15.28)$ & .003 \\
\hline 40 or older & 3810 & $1407(36.93)$ & $\operatorname{Ref}^{b}$ & $301(7.90)$ & $\operatorname{Ref}^{b}$ \\
\hline \multicolumn{6}{|l|}{ NHBS $^{d}$ city resident } \\
\hline Yes & 3673 & $1740(47.37)$ & $<.001$ & $482(13.12)$ & $<.001$ \\
\hline No & 5412 & $1862(34.41)$ & $\operatorname{Ref}^{b}$ & $435(8.04)$ & $\operatorname{Ref}^{b}$ \\
\hline \multicolumn{6}{|l|}{ Recruitment type } \\
\hline Gay social networking & 1268 & $416(32.81)$ & .005 & $74(5.84)$ & .009 \\
\hline General gay interest & 102 & $42(41.18)$ & .92 & $9(8.82)$ & .92 \\
\hline
\end{tabular}




\begin{tabular}{|c|c|c|c|c|c|}
\hline \multirow[t]{3}{*}{ Participant characteristics } & \multirow[t]{3}{*}{ Participants (N) } & \multicolumn{4}{|c|}{$\mathrm{STI}^{\mathrm{a}}$ history in the past 12 months } \\
\hline & & \multicolumn{2}{|c|}{ Tested for any STI } & \multicolumn{2}{|c|}{ Diagnosed with any STI } \\
\hline & & $\mathrm{n}(\%)$ & $P$ value $^{\mathrm{b}}$ & $\mathrm{n}(\%)$ & $P$ value ${ }^{\mathrm{b}}$ \\
\hline General social networking & 4022 & $134(33.37)$ & $\operatorname{Ref}^{b}$ & $297(7.38)$ & $\operatorname{Ref}^{\mathrm{b}}$ \\
\hline $\begin{array}{l}\text { Geospatial social network- } \\
\text { ing }\end{array}$ & 3688 & $1799(48.78)$ & $<.001$ & $537(14.56)$ & $<.001$ \\
\hline
\end{tabular}

${ }^{a}$ STI: sexually transmitted infection (includes chlamydia, gonorrhea, and syphilis).

${ }^{\text {b}}$ Wald chi-square $P$ values from the multivariate logistic regression model comparing behavior (yes vs no) between groups with specific characteristics and a reference (Ref) group.

${ }^{\mathrm{c}}$ Wald chi-square $P$ values from the multivariate logistic regression model comparing behavior (yes vs no) among HIV-positive participants and HIV-negative or unknown-serostatus participants. Model controlled for race/ethnicity, age, NHBS system city residency, and recruitment type.

d NHBS: National HIV Behavioral Surveillance.

\section{Acknowledgments}

The study was funded by a grant from the MAC AIDS Fund and by the National Institutes of Health (P30AI050409) — the Emory Center for AIDS Research.

\section{Conflicts of Interest}

TS and PS are members of the Editorial Board of JMIR Public Health and Surveillance. However, they had no involvement in the editorial decision for this manuscript. It was reviewed and handled by an independent editor.

\section{Multimedia Appendix 1}

American Men's Internet Survey 2017 questionnaire.

[DOCX File, 357 KB-Multimedia Appendix 1]

\section{References}

1. Finlayson TJ, Le B, Smith A, Bowles K, Cribbin M, Miles I, Centers for Disease Control and Prevention (CDC). HIV risk, prevention, and testing behaviors among men who have sex with men--National HIV Behavioral Surveillance System, 21 U.S. cities, United States, 2008. MMWR Surveill Summ 2011 Oct 28;60(14):1-34 [FREE Full text] [Medline: 22031280]

2. Sanchez TH, Sineath RC, Kahle EM, Tregear SJ, Sullivan PS. The Annual American Men's Internet Survey of behaviors of men who have sex with men in the United States: protocol and key indicators report 2013. JMIR Public Health Surveill 2015;1(1):e3 [FREE Full text] [doi: 10.2196/publichealth.4314] [Medline: 27227126]

3. Sanchez T, Zlotorzynska M, Sineath C, Kahle E, Sullivan P. The Annual American Men's Internet Survey of behaviors of men who have sex with men in the United States: 2014 Key Indicators Report. JMIR Public Health Surveill 2016 May 25;2(1):e23 [FREE Full text] [doi: 10.2196/publichealth.5476] [Medline: 27244770]

4. Zlotorzynska M, Sullivan P, Sanchez T. The Annual American Men's Internet Survey of behaviors of men who have sex with men in the United States: 2015 Key Indicators Report. JMIR Public Health Surveill 2017 Mar 25;3(1):e13 [FREE Full text] [doi: 10.2196/publichealth.7119] [Medline: 28356240]

5. Zlotorzynska M, Sullivan P, Sanchez T. The Annual American Men's Internet Survey of behaviors of men who have sex with men in the United States: 2016 Key Indicators Report. JMIR Public Health Surveill 2019 Feb 20;5(1):e11313 [FREE Full text] [doi: $10.2196 / 11313]$ [Medline: $\underline{\text { 30785405] }}$

6. Sanchez TH, Zlotorzynska M, Sineath RC, Kahle E, Tregear S, Sullivan PS. National trends in sexual behavior, substance use and HIV testing among United States men who have sex with men recruited online, 2013 through 2017. AIDS Behav 2018 Aug;22(8):2413-2425. [doi: 10.1007/s10461-018-2168-4] [Medline: 29948340]

7. Office of Policy Development and Research (PD\&R), US Department of Housing and Urban Development. HUD USPS ZIP code crosswalk files URL: https://www.huduser.gov/portal/datasets/usps_crosswalk.html [accessed 2017-02-15]

8. USPS. ZIP code lookup URL: https://tools.usps.com/go/ZipLookupAction input [accessed 2017-02-15]

9. Centers for Disease Control and Prevention. 2016 Jan. HIV Infection Risk, Prevention, and Testing Behaviors among Men Who Have Sex With Men-National HIV Behavioral Surveillance, 20 U.S. Cities, 2014. HIV Surveillance Special Report 15 URL: https://www.cdc.gov/hiv/pdf/library/reports/surveillance/cdc-hiv-hssr-nhbs-msm-2014.pdf [accessed 2019-10-15]

10. Ingram DD, Franco SJ. 2013 NCHS urban-rural classification scheme for counties. Vital Health Stat 2 2014 Apr(166):1-73 [FREE Full text] [Medline: 24776070] 


\section{Abbreviations}

AMIS: American Men's Internet Survey

MSM: men who have sex with men

NHBS: National HIV Behavioral Surveillance System

STI: sexually transmitted infection

ZIP: zone improvement plan

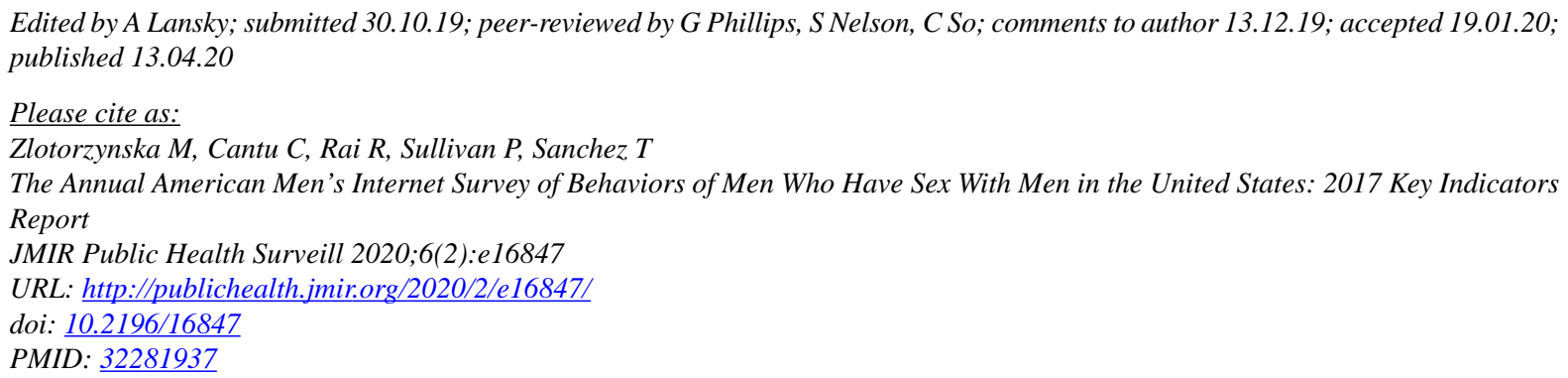

(CMaria Zlotorzynska, Cera Cantu, Ramona Rai, Patrick Sullivan, Travis Sanchez. Originally published in JMIR Public Health and Surveillance (http://publichealth.jmir.org), 13.04.2020. This is an open-access article distributed under the terms of the Creative Commons Attribution License (https://creativecommons.org/licenses/by/4.0/), which permits unrestricted use, distribution, and reproduction in any medium, provided the original work, first published in JMIR Public Health and Surveillance, is properly cited. The complete bibliographic information, a link to the original publication on http://publichealth.jmir.org, as well as this copyright and license information must be included. 